able to walk farther. The pills had acted well. The difference between the tracings of the first and second visit is sufficiently evident.

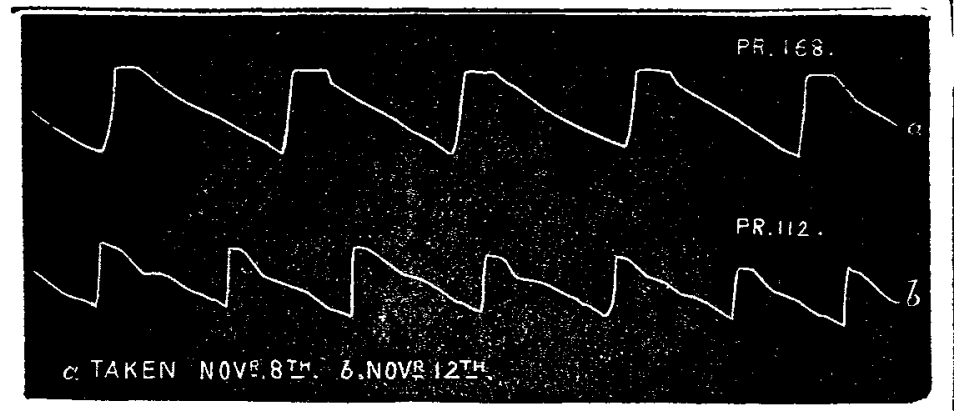

No doubt can exist that the sphygmograph was-here of practical utility. By demonstrating the existence of high fension in the arteries it suggested the true cause of the breathlessness and means for its speedy relief. The undue tension was probably produced by unbealthy matters in the blood; and the evacuation of these by oholagogue aperients enabled the blood to pass more freely through the capillaries, according to the view so ably maintained by Dr. Mahomed in his paper in the Medico-Chirurgical Trans. actions, vol. lvii.

CAse 8.-F. O-, aged fifty-two, a strongly-built, darkcomplexioned male, inclined to obesity. Weight nearly fourteen stone. Hair not lost. Was under my care ten or twelve years ago suffering with various neurotic disord-rs, apparently from being overworked. He used then to be very drowsy. His father died at the age of eighty-one. One brother has died of Bright's disease, and one of epileptic fits or softening of the brain (he used to take much morphia). He has had much mental anxiety and worry of late years. In June, 1875 , he bad an attack in which he was not unconscious, but spoke thick as if intoxicated; and this impairment of utterance continues, and his memory is defective. He speaks freely and rapidly; there is no defect in ideation, but only in phonation. There was no paralysis in, nor has there been after, the attack, but for some time, when he wrote, his right hand seemed to run away from him. Before the attack he had much giddiness, and this continues to trouble him. His vision is very defective unless he uses glasses, of which he has two or three for various distances. He is a plumber, but has done no manual work for many years; has no blue line. Urine of good colour, sp.gr. 1022, clear, not albuminous, deposits notbing but a little oxalate of lime. Tongue rather coated at back. Bowels regular. Is subject to palpitation of heart; does not know always what brings it on. Cardiac impulse felt in epigastrium; nowhere else; first sound appears normal, second is greatly prolonged, almost amounting to a murmur, at mid-sternum, at xiphoid, and at second right cartilage. Going upstairs distresses him much, often brings on palpitation, puts him out of breath. No râles in lungs. Perspires freely, especially about the head. Is better in cold, frosty weather. Has a deposit of about the size of a pea-rather like a gouty-in the left forefinger; has had pains in his toes and fingers occasionally, but no regular attack of gout.

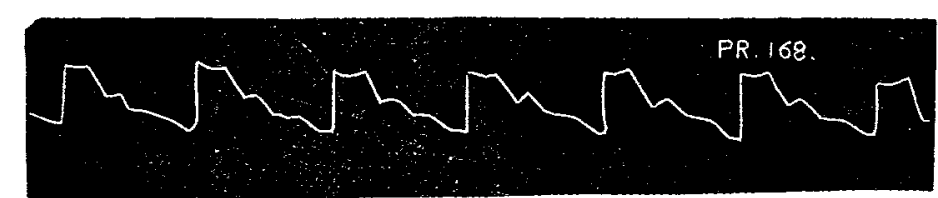

Here the giddiness, the impairment of speech, and the death of a brother with brain symptoms, might have sug. gested the possible existence of some morbid change in the cerebral arterioles; but the palpitation of the heart, the breathlessness, and the remarkable prolongation of the second sound, especially in the absence of signs of morbus Brightii, would have remained unexplained. The demonstration of rigid arteries by the spbygmograph both furnished a solid basis for the bypothesis of obstructed small vessels within the head and assigned a cause for the cardiac symptoms. Without the aid afforded by the tracing I am sure I should have been much at fault. The death of a brother from morbus Brightii is another instance of the a finity between renal degeneration and that of the arterial system generally. One point in the above history, and in another similar which $I$ possess, is difficult of explanation- namely, that the patients were better in cold weather. One would have thought that the cold, by contracting the arteries still further, would have made the circulation of blood more difficult. Perhaps the heart's tissue may have been more braced by the cold than the arteries were con. tracted, so that the total result was good.

(To be concluded.)

\section{ON ORCHITIS.}

\section{B Y J O H N G A $\mathrm{Y}$, E. R. C. S.,} SURGEON TO THE GREAT NORTHERK BOSPITAL.

I SHOULD be glad to be allowed, whilst the subject of orchitis is on the tapis, to call the attention of the profession to a few points which have, I think, been overlooked in the controversy that has been going on in the columns of THE LANCeT. Several apparently conflicting statements have been made with regard to the treatment of orchitis, each in advocady of some especial remedy; and the logical inference is: that dither all must be right; or all but one must be wrong. My friend, Mr. Henry Smith, avers that he has treated over a thousand cases of that disease with saccess by incieion or puneture inte the body of the testis-a method attributed to Vidal de Cassis; others affirm that they have obtained like favourable results by emptying the tunica vaginalis of some serum effused in the course of the disorder; whilst a third class commends rest, and, at the utmost, a few leeches, as all that is necessary to the like result. It is not credible that observers of either class can have made their statements respectively without some real and indisputable foundation for them. The slit of Vidal must have been followed in some instances by relief-I have had no experience of it,-as well as tapping the vaginal sac, and antiphlogistics. On the other hand, there can be as little doubt that each remedy has likewise signally failed. This reduces the treatment of orchitis to something very akin to charlatinism, if by this term we mean to imply something that is speculative, in contradistinction to that which is based upon an observance of the inevitable laws of anatcmical and pathological science. And the only course that a poor wreteh, who is suffering under such a painful malady, can therefore reasonably pursue is to take the round of the three theorists, with an assurance that he will sooner or later get the relief that he seeks.

Now, something must be wrong here; and I would ven. ture to suggest that the apparent contradictions by men of established reputation may be reconciled on the gra. tuitous hypothesis that the cases spoken of as having been subjected to treatment belonged to different forms of orchitis, and could not therefore be expected to be amenable to one and the same class of remedies. The word "orchitis" has been used; but, on referring to my case-kook (for I have long taken an interest in this disorder), I find as many varieties as there are of inflammation of any other organ, if not more. I have dotted down cases of trasmatic, as well as of idiopathic, rheumatic and perbaps gouty, and sympathetic orchitis; the latter variety being further subdivided into orehitis associated with other glandular inflammations, especially the parotid, with gonorrhcea, and with urethral stricture. I find, too, that these forms of the affection have certain pathological distinctions, which have been found by reference and experience to bring each into relation with certain therapeutical agents almost as specifically as scabies to sulphur; it may be a puncture in one form, leeches and tartar emetic in a second, strapping or cotton-wool (as recommended by Songet) in a third, iodide of potassium in a fourth, or narcotics in a fifth.

Vidal, it must be remembered, advised puncture or "débridement" in cases of gonorrboeal orchitis only; and these he divided into "orchite parenchymateuse," "vaginalité," and "epididymité," recommending the operation (if it may be so called) in each form alike. In the former, however, he only designed to divide the tunica albuginea, and that to the extent of a centimetre and a half, and this in order to relieve the " entranglement" of its contents. It is possible, he says, that even with the precaution he insists 
upon, the seminal tubes may be wounded, but denies that such an occurrence could in any way injure the organ.

It is hard to understand how this slit can relieve the inflamed parenchyma of the testis, except by either allowing hernia of the tubuli or the exudation of inflammatory products. And this last explanation is by no means improbable, especially in the advance upon Vidal's plan, which seems to consist in carrying the puncture or incision into the tubular elements. The microscope would throw interesting light on this part of the subject, and should be used.

As I have hinted, the readers of THE LANCET have been left in the dark as to the specific form of orchitis in which incision has been so successfully performed; and, from the turn the discussion has taken, are led to suppose that this and rival remedies have been used some what promiscuously, at all events so much so as to leave the question at issue, whether incision should be looked upon as one of the remedies for orchitis, in a state of indecision.

This view zeems to me to remove any solid basis for criticising this method of treatment; for surely distinct varieties of this affection cannot be expected to yield to one uniform mode of treatment-i.e., what may be good for one is not good for another; and each may be good if adopted on the safer grounds of pathological and therapeutical indications.

It may be that gonorrhœal orchitis has been most prominently in the minds of the gentlemen who bave contri buted to the discussion, since this is by far the most common form, and that to which the greatest attention has been given, and for which the greatest number of curative resources has been suggested; and with this impression, with out attempting, beyond what I have said, to help in the settlement of the points raised, $I$ beg to call the attention of the profession to a method of treatment which (although from the omission of all reference to it in moderu works it has seemed to have become generally obsolete) has been in my hands, and in the hands of others, eminently successfu in these cases, by rapidly assuaging the pain attendant on this disease, and apparently inducing early reaction in the direction of resolution. I refer to treatment based on the observation of facts which go to show that in by far the greater number of instances the infammatory action in gonorrbcal orchitis is purely of an asthenic character, and due to its propagation either directly from the urethra, where it simply languisbes through its submucous cellular tissue, to the connective tissue of the seminal tubules; or by sympathetic agency alone withont intervening physical media. I am inclined to adopt the latter explanation, and to regard the disorder, especially in its metastatic form, as akin to pyamia.

In the number of THe lancet for August 10th, 1844, I bronght forward a number of cases from my hospital practice which showed that narcotics were in the highest degree serviceable in this disease by first reducing what was then called the "morbid sensibility," now known by the more classic and euphonious name of "byperæsthesia" of the inflamed tissues. As soon as this result was attained the tence condition of the testicle and its coverings generally yielded, and the disorder went on towards resolution, with or without effusion into the tunica vaginalis, in a most satisfactory manner.

This paper was reviewed in the Medico-Chimurgical Review for Oct. 1st, 18t4. The practice was publicly approved by many who tried it; amongst others, by my friends Mr. T. Carr Jackson, who illu e aied its advantages by a recital of successful cases in THE LANCET for 1848 , vol, ii., p. 338, Mr. Milton, in his work "On Gonorrbca"; and by dMr. Childs.

I referred to the disease at that time in the following manner:- "The variety of orchitis to which I refer is that commonly met with after the fourth week of gonorrhoal urethritis, and is marked by the following symptomsgeneral debility, unusual palor of the face and mucous membranes, skin perspiring on the least exertion, quick pulse, foul tongue, high-coloured urine, and constipation. There is dull, sching pain about the testis and epididymis, increased by pressure and exertion, followed often by the formation of a dense tumour, generally in the most de. pending part of the globus minor, and circumseribed by its investing tunic. The inflammatory action usually extends to the testis, the vas deferens, the general tissues of the cord, which become swollen and cedicmatous, and the scrotum, the veing of which of ten show extreme turgescence; so that in extreme cases the whole organ and its investments become involved in one hard, painful mass."

I had an opportunity of examining a case in a man who was killed by an accident whilst suffering from a sharp attack. The inflammation attacked in order the epididymis, testis, cord, and scrotum. He was under my observation several days before death. The parts-were all very much swollen up to the ring. The scrotal veins were exceedingly distended; the testis and epididymis, on being cut through, exhibited a roseate hue and a vast number of minute vascular surfaces over the entire cut surfaces; the globus minor and most depending portion of the testis were, by a shade, of a deeper red than the other parts, and slightly more dense, the structure in these parts being more distinct through effasion between the tubuli; the tubuli themselves were patent but compressed; there was a little fluid, not much more than in health, in the tunica vaginalis; the sheath and tissues of the cord were excessively distended by venous blood and serum up to the external abdowinal ring, through constriction by its edges; above the ring the cord was not above its normal size; the vas deferens was thickened and dense; the mucous membrane of the urethra was throughont velvety and vascular, and mottled by groups of large bloodvessels, apparently veins.

The details of treatment which 'I proposed, and which succeeded admirably in a kundred recorded cases, were as follows:-Rest on the back, with cold applications; a good purge, with five grains of calomel, repeated once and again if necessary, and a drachm of tincture of henbane three times a day until the patient was brought obviously under its influence, - a larger dose if necessary; opening a vein or two in the scrotum, provided the abdominal ring compressed the cord so as to occasion scrotal congestion. On the subsidence of the pain, quinine or steel, or both combined, and an injection of sulphate of zinc or acetate of lead, in solution, into the urethra. The use of a small trocar or grooved needle as soon as fluid is felt in the tunica vaginalis.

As an injection, I now use a dilution of the solution of pernitrate of mexcury; but it must be used very cautiously, beginning with two or four minims to eight ounces of water, and gradually increasing it until it is sufficiently strong to give a little uneasiness or pain.

Rest in these cases is very effective; but I believe the treatment described in addition will prove to be an exceedingly valuable adjunct, and all that is needed for the greater number of cases of gonorrhcal orchitis.

Finsburg-place Soutl.

\section{A NEW METHOD OF FREEZING TISSUES FOR THE MICROSCOPE.}

\section{BY CHARLES COPPINGER,}

ASSIETANT-BCRGZON TO THE MATER MISERICORDIE HOSPITAL.

IN a recent number of THE LANCET there appear a description and illustration of an ingenious apparatus devised by Dr. Urban Pitehard for freezing tissues for microscopic purposes.

I have been for some years making use of a somewhat novel method of bringing about the same result, and I hope that its simplicity will recommend it to the readers of THe LANCET. There is no doubt that of all the methods of hardening tissues to erable thin sections to be made of them, there is none so satisfactory as that, of freezing, provided the mechavical difficulties in connexion with the use of ice were removed.

The freezing method is especially suitable for the immediate examination of recent pathologicul tissues, but unfortunately it is just in these cases that the difficulty of obtaining ice most of ten meets one. Under such circumstances I have been in the habit fur a long time of freezing the tissue by mears of the ether spray.

At first the process consisted simply in directing the spray against a small piece of the fresh structure hid on the end of an oroinary cork. In four or five seconds it is thus frozen to the waxy condition suitable for catting. A 